\title{
DIRECT INTEGRALS OF MATRICES
}

\author{
PIOTR NIEMIEC
}

\begin{abstract}
It is shown that each linear operator on a separable Hilbert space which generates a finite type I von Neumann algebra has, up to unitary equivalence, a unique representation as a direct integral of inflations of mutually unitary inequivalent irreducible matrices. This leads to a simplification of the so-called prime (or central) decomposition and the multiplicity theory for such operators. The concept of so-called p-isomorphisms between special classes of such operators is discussed. All results are formulated in more general settings; that is, for tuples of closed densely defined operators affiliated with finite type I von Neumann algebras.
\end{abstract}

\section{INTRODUCTION}

In [6] Ernest proved that every bounded linear operator acting on a separable Hilbert space has, up to unitary equivalence, a unique so-called central decomposition. His definition of this decomposition involved the reduction theory of von Neumann algebras (due to von Neumann himself [13]). More precisely, the direct integral

$$
T=\int^{\oplus} T_{\omega} \mathrm{d} \mu(\omega)
$$

is the central decomposition of $T$ iff $\int^{\oplus} \mathcal{W}\left(T_{\omega}\right) \mathrm{d} \mu(\omega)$ is the central decomposition of $\mathcal{W}(T)$ where for any bounded linear operator $S, \mathcal{W}(S)$ denotes the smallest von Neumann algebra containing $S$. Recently 10 we generalised Ernest's theorem to the context of finite tuples of closed densely defined linear operators acting in a common (totally arbitrary, possibly nonseparable) Hilbert space. We call our result the Prime Decomposition Theorem (see Chapter 5 in 10 and Theorem 5.6.14 there). In practice it is not so easy to resolve whether the direct integral of the form (1-1) is the central decomposition of a separable Hilbert space operator T. A necessary condition for this is that off some null set, $\mathcal{W}\left(T_{\omega}\right)$ is a factor and the operators $T_{\omega}$ and $T_{\omega^{\prime}}$ for distinct $\omega$ and $\omega^{\prime}$ have no nontrivial unitary equivalent suboperators (in that case we call the operators $T_{\omega}$ and $T_{\omega^{\prime}}$ unitarily disjoint). The main disadvantage of both the results cited above is that the last mentioned condition is insufficient in general for (1-1) to be the central decomposition of $T$. So, some bounded operators $T$ admit two inequivalent representations as direct integrals of pairwise unitarily disjoint factor operators. However, as we will show in this paper, if $T$ generates a finite type I von Neumann algebra, its central decomposition may simply be recognised: in that case, a necessary and sufficient condition for (1-1) to be the central decomposition of $T$ is that off some null set, the field $\left\{T_{\omega}\right\}$ consists of inflations of pairwise unitarily inequivalent irreducible matrices, as shown by

\footnotetext{
2010 Mathematics Subject Classification. Primary 47B40; Secondary 47C15.

Key words and phrases. Direct integral; finite type I von Neumann algebra; central decomposition of an operator; prime decomposition of a tuple of operators; spectral theorem; multiplicity

The author gratefully acknowledges the assistance of the Polish Ministry of Sciences and Higher Education grant NN201 546438 for the years 2010-2013.
} theory. 
1.1. Theorem. (a) For $j \in\{1,2\}$, let $\left\{T_{j}\left(\omega_{j}\right)\right\}_{\omega_{j} \in \Omega_{j}}$ be a measurable field defined on a standard measure space $\left(\Omega_{j}, \mathfrak{M}_{j}, \mu_{j}\right)$ such that off some $\mu_{j}$-null set, the operators $T_{j}\left(\omega_{j}\right)$ are unitarily equivalent to inflations of pairwise unitarily inequivalent irreducible matrices. Then

$$
\int_{\Omega_{1}}^{\oplus} T_{1}\left(\omega_{1}\right) \mathrm{d} \mu_{1}\left(\omega_{1}\right) \text { and } \int_{\Omega_{2}}^{\oplus} T_{2}\left(\omega_{2}\right) \mathrm{d} \mu_{2}\left(\omega_{2}\right) \text { are unitarily equivalent }
$$

iff there are measurable sets $Z_{1} \subset \Omega_{1}$ and $Z_{2} \subset \Omega_{2}$, and a Borel isomorphism $\Phi: \Omega_{1} \backslash Z_{1} \rightarrow \Omega_{2} \backslash Z_{2}$ such that:

- $\mu_{1}\left(Z_{1}\right)=\mu_{2}\left(Z_{2}\right)=0$;

- for any measurable set $B \subset \Omega_{1} \backslash Z_{1}, \mu_{1}(B)=0$ if and only if $\mu_{2}(\Phi(B))=0$;

- the operators $T_{2}\left(\Phi\left(\omega_{1}\right)\right)$ and $T_{1}\left(\omega_{1}\right)$ are unitarily equivalent for any $\omega_{1} \in$ $\Omega_{1} \backslash Z_{1}$.

(b) Let $T$ be a closed densely defined linear operator in a separable Hilbert space. Then $T$ is unitarily equivalent to a decomposable operator of the form

$$
\int_{\Omega}^{\oplus} T(\omega) \mathrm{d} \mu(\omega)
$$

where $\{T(\omega)\}_{\omega \in \Omega}$ is a measurable field defined on a standard measure space $(\Omega, \mathfrak{M}, \mu)$ such that the operators $T(\omega)$ are unitarily equivalent to inflations of pairwise unitarily inequivalent irreducible matrices iff $T$ is affiliated with a finite type I von Neumann algebra.

A certain generalisation of the above theorem (which will be stated and proved in Section 3) shall be applied in the sequel to prove that certain classes of tuples of operators are p-isomorphic. Roughly speaking, the existence of a p-isomorphism between two classes asserts that these classes have the same Borel (as well as spectral) complexity (p-isomorphisms preserve direct sums of arbitrary collections and prime decompositions). As a main result in this direction we will obtain a theorem stating that the class of all $k$-tuples (where $k$ is arbitrarily fixed) of closed densely defined linear operators affiliated with finite type I von Neumann algebras is p-isomorphic to the class of all (single) unitary operators.

The paper is organised as follows. In Section 2 we study finite Borel measures on sets of tuples of matrices. The main property established there is used in Section 3 to prove Theorem 1.1 and its certain generalisation to finite tuples of operators (Theorem 3.1 below). The last, fourth, part is devoted to so-called finite type I ideals of unitary equivalence classes of tuples of operators. We recall there our Prime Decomposition Theorem from [10] and prove that some finite type I ideals are p-isomorphic. We conclude the paper with a concept of multiplicity theory for finite tuples of operators which are affiliated with finite type I von Neumann algebras.

Notation and terminology. All Hilbert spaces (as well as matrices) are complex. An operator means a closed linear operator densely defined in a Hilbert space. Each $k$-tuple of operators (resp. matrices) consists of operators acting in a common, but arbitrary, Hilbert space (resp. of square matrices whose degrees coincide). For a $k$-tuple $\left(T_{1}, \ldots, T_{k}\right)$ of bounded operators, we denote by $\mathcal{W}\left(T_{1}, \ldots, T_{k}\right)$ the smallest von Neumann algebra which contains each of $T_{1}, \ldots, T_{k}$. If $T_{1}, \ldots, T_{k}$ are merely closed and densely defined, let $\mathcal{W}^{\prime}\left(T_{1}, \ldots, T_{k}\right)$ stand for the von Neumann algebra generated by all unitary operators $U$ for which $U T_{j} U^{-1}=T_{j}(j=1, \ldots, k)$. Additionally, we put $\mathcal{W}^{\prime \prime}\left(T_{1}, \ldots, T_{k}\right)=\left(\mathcal{W}^{\prime}\left(T_{1}, \ldots, T_{k}\right)\right)^{\prime}$. Recall that $\mathcal{W}^{\prime \prime}\left(T_{1}, \ldots, T_{k}\right)=$ $\mathcal{W}\left(T_{1}, \ldots, T_{k}\right)$ provided $T_{1}, \ldots, T_{k}$ are bounded. The $k$-tuple $\left(T_{1}, \ldots, T_{k}\right)$ is said to be irreducible if $\mathcal{W}^{\prime}\left(T_{1}, \ldots, T_{k}\right)$ consists (precisely) of scalar multiples of the identity operator. Two $k$-tuples $\left(T_{1}, \ldots, T_{k}\right)$ and $\left(T_{1}^{\prime}, \ldots, T_{k}^{\prime}\right)$ are unitarily equivalent if 
there exists a single unitary operator $U$ (between respective Hilbert spaces) such that $U T_{s} U^{-1}=T_{s}^{\prime}$ for each $s \in\{1, \ldots, k\}$. By an inflation of $\left(T_{1}, \ldots, T_{k}\right)$ we mean a $k$-tuple of the form $\left(\bigoplus_{s \in S} T_{1}^{(s)}, \ldots, \bigoplus_{s \in S} T_{k}^{(s)}\right)$ where $S$ is a nonempty set and $T_{j}^{(s)}=T_{j}$ for any $s \in S$ and $j \in\{1, \ldots, k\}$ (with this terminology we follow e.g. Conway [4] - see Example 5.3 there). The $\mathfrak{b}$-transform (cf. [10]) of an operator $T$ is given by $\mathfrak{b}(T) \stackrel{\text { def }}{=} T(I+|T|)^{-1}$ where $|T|=\left(T^{*} T\right)^{\frac{1}{2}}$ is the absolute value of $T$ and $I$ is the identity operator on a respective Hilbert space. Whenever $X$ is a topological space, $\mathfrak{B}(X)$ denotes the $\sigma$-algebra of all Borel sets in $X$; that is, $\mathfrak{B}(X)$ is the smallest $\sigma$-algebra which contains all open subsets of $X$. A map is a continuous function.

For an easier understanding of the paper, it is recommended to study treatises [10] and 6].

\section{MeAsures on SETS OF TUPLES OF MATRICES}

For each $n \geqslant 1$ denote by $\mathscr{M}_{n}$ the $C^{*}$-algebra of all $n \times n$ matrices and by $\mathscr{U}_{n}$ the unitary group of $\mathscr{M}_{n}$ (with unit $I$ ). Fix $k \geqslant 1$ (as the length of tuples) and equip the vector space $\mathscr{M}_{n}^{k}$ of all $k$-tuples of $n \times n$ matrices with the action of $\mathscr{U}_{n}$ given by

$$
U .\left(A_{1}, \ldots, A_{k}\right)=\left(U A_{1} U^{-1}, \ldots, U A_{k} U^{-1}\right) \quad\left(U \in \mathscr{U}_{n},\left(A_{1}, \ldots, A_{k}\right) \in \mathscr{M}_{n}^{k}\right) .
$$

Since our main interest are irreducible $k$-tuples, the following simple lemma may be helpful in understanding the notion introduced above. We leave its proof to the reader (use the fact that $\mathcal{W}^{\prime}\left(A_{1}, \ldots, A_{k}\right)$ is a von Neumann algebra).

2.1. Lemma. A tuple $\left(A_{1}, \ldots, A_{k}\right) \in \mathscr{M}_{n}^{k}$ is irreducible iff for any $U \in \mathscr{U}_{n}$,

$$
U .\left(A_{1}, \ldots, A_{k}\right)=\left(A_{1}, \ldots, A_{k}\right) \Longleftrightarrow U=\gamma \cdot I \text { for some } \gamma \in \mathbb{C} \text {. }
$$

We use $\mathscr{I}_{n}(k)$ to denote the set of all irreducible $k$-tuples of $n \times n$ matrices. It is well-known that $\mathscr{I}_{n}(k)$ is an open set in $\mathscr{M}_{n}^{k}$ (hence $\mathscr{I}_{n}(k)$ is a separable locally compact space). Observe also that $\mathscr{I}_{n}(k)$ is invariant under the action of $\mathscr{U}_{n}$. We now introduce

2.2. Definition. Let $\mathfrak{I}_{n}(k)$ stand for the orbit space of the action of $\mathscr{U}_{n}$ on $\mathscr{I}_{n}(k)$; that is, $\mathfrak{I}_{n}(k)$ consists of all sets of the form $\left\{U .\left(A_{1}, \ldots, A_{k}\right): U \in \mathscr{U}_{n}\right\}$ where $\left(A_{1}, \ldots, A_{k}\right) \in \mathscr{I}_{n}(k)$. Further, let $\pi_{n}^{k}: \mathscr{I}_{n}(k) \rightarrow \mathfrak{I}_{n}(k)$ be the natural projection. We equip $\mathfrak{I}_{n}(k)$ with the quotient topology.

Since the group $\mathscr{U}_{n}$ is compact and $\mathscr{I}_{n}(k)$ is locally compact, it is easily seen that $\mathfrak{I}_{n}(k)$ is a separable metrizable locally compact space and $\pi_{n}^{k}$ is a proper map. What is more,

2.3. Proposition. There exists a $\mathcal{G}_{\delta}$-set $\mathscr{S}_{n}(k) \subset \mathscr{I}_{n}(k)$ which meets the orbit of each irreducible $k$-tuple at exactly one point; in other words, the restriction of $\pi_{n}^{k}$ to $\mathscr{S}_{n}(k)$ is a Borel isomorphism of $\mathscr{S}_{n}(k)$ onto $\mathfrak{I}_{n}(k)$.

The above result may be deduced e.g. from Corollary 1 in $\S 2$ of Chapter XIV in 8], applied to the partition $\mathfrak{I}_{n}(k)$ of $\mathscr{I}_{n}(k)$, or from Corollary 1 in $\S 1$ of Chapter XIV in [8] (see also [3]; for more information on selection theorems consult Chapter XIV of [8] or $\S 12 . \mathrm{D}$ of Chapter II in []).

To simplify further statements, we fix $k>0$ and for any $n>0$ a set $\mathscr{S}_{n}(k) \subset$ $\mathscr{I}_{n}(k)$ which witnesses the assertion of Proposition 2.3, and consider the topological disjoint union $\mathscr{S}(k)=\bigsqcup_{n=1}^{\infty} \mathscr{S}_{n}(k)$ of the topological spaces $\mathscr{S}_{n}(k), n>0$. Note that $\mathscr{S}(k)$, as a topological disjoint union of $\mathcal{G}_{\delta}$-sets, is separable and completely metrizable. The aim of this section is to show that all $\sigma$-finite Borel measures on $\mathscr{S}(k)$ are supported on certain sets, which we now introduce. 
2.4. Definition. A set $\mathscr{F}$ of $k$-tuples of matrices is said to be a measurable domain of strong unitary disjointness (or, briefly, a measurable domain) if $\mathscr{F} \cap \mathscr{M}_{n}^{k}$ is Borel for any $n>0$ and there is a countable collection $\left\{F_{1}, F_{2}, \ldots\right\}$ of $k$-tuples of matrices which separates points of $\mathscr{F}$ (that is, for any two distinct tuples of $\mathscr{F}$ there is a number $s$ such that $F_{s}$ contains exactly one of them) and for any number $m$ there exists a sequence $p_{1}, p_{2}, \ldots$ of complex polynomials in $2 k$ noncommuting variables such that the matrices $p_{n}\left(\mathfrak{b}\left(X_{1}\right), \ldots, \mathfrak{b}\left(X_{k}\right), \mathfrak{b}\left(X_{1}\right)^{*}, \ldots, \mathfrak{b}\left(X_{k}\right)^{*}\right)$ converge to 0 for any $\left(X_{1}, \ldots, X_{k}\right) \in \mathscr{F} \backslash F_{m}$ and to a respective unit matrix for any $\left(X_{1}, \ldots, X_{k}\right) \in$ $\mathscr{F} \cap F_{m}$.

Measurable domains were introduced in [10] and used to characterise so-called prime decompositions of tuples of operators. A more detailed explanation of this will be given in Theorem 4.6 (consult also Theorem 4.5 in Section 4).

The aim of this part is to prove

2.5. Theorem. Every $\sigma$-finite Borel measure on $\mathscr{S}(k)$ is supported on a measurable domain.

The proof of the above theorem shall be preceded by a few auxiliary results. The first of them is a kind of folklore. Since we could not find it in the literature, we sketch its simple proof.

2.6. Lemma. If $\mu$ is a finite Borel measure on a separable complete metric space $X$ and $B$ is a Borel set in $X$, then for every $\varepsilon>0$ there is a totally disconnected compact set $K \subset B$ such that $\mu(B \backslash K) \leqslant \varepsilon$.

Proof. It is well-known that finite Borel measures on separable complete metric spaces are regular; that is, $\mu(C)=\sup \{\mu(L): L \subset C, L$ compact $\}$ for each Borel set $C$. Since for any $\delta>0$ the set $B$ may be covered by a countable number of pairwise disjoint Borel sets whose diameters are less than $\delta$, we infer from the regularity of $\mu$ that for any $s>0$, there is a finite collection $\left\{K_{1}^{(s)}, \ldots, K_{p_{s}}^{(s)}\right\}$ of pairwise disjoint compact subsets of $B$ whose diameters are less than $\frac{1}{s}$ such that $\mu\left(B \backslash \bigcup_{n=1}^{p_{s}} K_{n}^{(s)}\right) \leqslant \frac{\varepsilon}{2^{s}}$. Then the set $K=\bigcap_{s=1}^{\infty}\left(\bigcup_{n=1}^{p_{s}} K_{n}^{(s)}\right)$ is totally disconnected and compact, and satisfies $\mu(B \backslash K) \leqslant \varepsilon$.

The following result may be seen as a special case of the factorial Stone-Weierstrass theorem due to Longo [9] and Popa [12].

2.7. Lemma. Let $\mathscr{K}$ be a compact subset of $\mathscr{S}(k) \cap \mathscr{M}_{n}^{k}$ for some $n>0$. Then the $C^{*}$-algebra $C\left(\mathscr{K}, \mathscr{M}_{n}\right)$ of all $\mathscr{M}_{n}$-valued continuous functions on $\mathscr{K}$ coincides with the smallest unital $C^{*}$-subalgebra of $C\left(\mathscr{K}, \mathscr{M}_{n}\right)$ which contains the maps $b_{j}: \mathscr{K} \ni$ $\left(X_{1}, \ldots, X_{k}\right) \mapsto \mathfrak{b}\left(X_{j}\right) \in \mathscr{M}_{n}(j=1, \ldots, k)$.

Proof. Denote by $\mathcal{E}$ a unital $C^{*}$-algebra generated by $\left\{b_{1}, \ldots, b_{k}\right\}$. Let $\left(X_{1}, \ldots, X_{k}\right)$ and $\left(Y_{1}, \ldots, Y_{k}\right)$ be two distinct members of $\mathscr{K}$. Since $\left(X_{1}, \ldots, X_{k}\right)$ is irreducible, so is $\left(\mathfrak{b}\left(X_{1}\right), \ldots, \mathfrak{b}\left(X_{k}\right)\right)$ and, consequently,

$$
\left\{f\left(X_{1}, \ldots, X_{k}\right): f \in \mathcal{E}\right\}=\mathscr{M}_{n} .
$$

Furthermore, since

$$
\pi_{n}^{k}\left(\mathfrak{b}\left(X_{1}\right), \ldots, \mathfrak{b}\left(X_{k}\right)\right) \neq \pi_{n}^{k}\left(\mathfrak{b}\left(Y_{1}\right), \ldots, \mathfrak{b}\left(Y_{k}\right)\right),
$$

the irreducibility of the $k$-tuples $\left(\mathfrak{b}\left(X_{1}\right), \ldots, \mathfrak{b}\left(X_{k}\right)\right)$ and $\left(\mathfrak{b}\left(Y_{1}\right), \ldots, \mathfrak{b}\left(Y_{k}\right)\right)$ is followed by the fact that there is a complex polynomial $p$ in $2 k$ noncommuting variables such that

$$
\begin{gathered}
p\left(\mathfrak{b}\left(X_{1}\right), \ldots, \mathfrak{b}\left(X_{k}\right), \mathfrak{b}\left(X_{1}\right)^{*}, \ldots, \mathfrak{b}\left(X_{k}\right)^{*}\right)=I, \\
p\left(\mathfrak{b}\left(Y_{1}\right), \ldots, \mathfrak{b}\left(Y_{k}\right), \mathfrak{b}\left(Y_{1}\right)^{*}, \ldots, \mathfrak{b}\left(Y_{k}\right)^{*}\right)=0
\end{gathered}
$$


(cf. e.g. [1]). So, for some $g \in \mathcal{E}$,

$$
g\left(X_{1}, \ldots, X_{k}\right)=I \quad \text { and } \quad g\left(Y_{1}, \ldots, Y_{k}\right)=0 .
$$

Now a combination of (2-1) and (2-2) implies that $\mathcal{E}=C^{*}\left(\mathscr{K}, \mathscr{M}_{n}\right)$, which follows directly from Proposition 2.3 in [11] or Corollary 11.5.3 in [5].

As a consequence, we obtain

2.8. Corollary. Let $\mathscr{K}$ be a totally disconnected and compact subset of $\mathscr{S}(k) \cap \mathscr{M}_{n}^{k}$ for some $n>0$. Then $\mathscr{K}$ is a measurable domain.

Proof. Let $\mathcal{F}$ be a collection of all clopen (that is, simultaneously open and closed) subsets of $\mathscr{K}$. Then $\mathcal{F}$ is countable (because $\mathscr{K}$ is metrizable and compact), separates points of $\mathscr{K}$ (by the total disconnectedness of $\mathscr{K}$ ) and, by Lemma 2.7 for any $F \in \mathcal{F}$, the function $j_{F}: \mathscr{K} \rightarrow \mathscr{M}_{n}$ given by

$$
j_{F}\left(X_{1}, \ldots, X_{k}\right)= \begin{cases}I & \left(X_{1}, \ldots, X_{k}\right) \in F, \\ 0 & \left(X_{1}, \ldots, X_{k}\right) \notin F\end{cases}
$$

belongs to the smallest unital $C^{*}$-algebra which contains the maps $b_{1}, \ldots, b_{k}$ introduced in Lemma 2.7. Consequently, for any $F \in \mathcal{F}$, there is a sequence $p_{1}, p_{2}, \ldots$ of complex polynomials in $2 k$ noncommuting variables such that

$$
\lim _{n \rightarrow \infty} p_{n}\left(\mathfrak{b}\left(X_{1}\right), \ldots, \mathfrak{b}\left(X_{k}\right), \mathfrak{b}\left(X_{1}\right)^{*}, \ldots, \mathfrak{b}\left(X_{k}\right)^{*}\right)=j_{F}\left(X_{1}, \ldots, X_{k}\right)
$$

for any $\left(X_{1}, \ldots, X_{k}\right) \in \mathscr{K}$ (and the convergence is uniform on $\mathscr{K}$ ). Now the very definition of a measurable domain yields the assertion.

Proof of Theorem 2.5. For the purpose of this proof, we follow some concepts of 10. In particular:

- we call a $\sigma$-finite Borel measure on $\mathscr{S}(k)$ a regularity measure if it is supported on a measurable domain;

- for two regularity measures $\mu$ and $\nu$ we write $\mu \perp_{s} \nu$ if $\mu$ and $\nu$ are mutually singular and $\mu+\nu$ is a regularity measure as well;

- two $k$-tuples $\left(T_{1}, \ldots, T_{k}\right)$ and $\left(T_{1}^{\prime}, \ldots, T_{k}^{\prime}\right)$ of operators are unitarily disjoint, in symbols $\left(T_{1}, \ldots, T_{k}\right) \perp_{u}\left(T_{1}^{\prime}, \ldots, T_{k}^{\prime}\right)$, if no nontrivial part of $\left(T_{1}, \ldots, T_{k}\right)$ is unitarily equivalent to a part of $\left(T_{1}^{\prime}, \ldots, T_{k}^{\prime}\right)$.

So, our aim is to show that in the above context the property of being a regularity measure is automatic.

Let $\mu$ be a $\sigma$-finite Borel measure on $\mathscr{S}(k)$. We may and do assume that $\mu$ is finite. It follows from Lemma 2.6 that there is a collection $\left\{\mathscr{K}_{n, m}: n, m>0\right\}$ of mutually disjoint totally disconnected compact sets such that for any $n>0$ :

- $\mathscr{K}_{n, m} \subset \mathscr{S}(k) \cap \mathscr{M}_{n}^{k}$ for any $m>0$;

- $\mu\left(\mathscr{S}(k) \cap \mathscr{M}_{n}^{k} \backslash \bigcup_{m=1}^{\infty} \mathscr{K}_{n, m}\right)=0$.

For simplicity, let $\mu_{n, k}$ be a Borel measure given by $\mu_{n, k}(\mathscr{B})=\mu\left(\mathscr{B} \cap \mathscr{K}_{n, m}\right)$. It follows from Corollary 2.8 that $\mu_{n, k}$ is a regularity measure. We shall now show that

$$
\mu_{n, m} \perp_{s} \mu_{n^{\prime}, m^{\prime}}
$$

for any two distinct pairs $(n, m)$ and $\left(n^{\prime}, m^{\prime}\right)$. To this end, we will separately deal with the cases when $n=n^{\prime}$ and $n \neq n^{\prime}$. In the former case, (2-3) follows immediately from Corollary 2.8 (because $K_{n, m} \cup K_{n, m^{\prime}}$ is totally disconnected). Now assume $n \neq n^{\prime}$ and consider $k$-tuples $\left(T_{1}, \ldots, T_{k}\right)$ and $\left(T_{1}^{\prime}, \ldots, T_{k}^{\prime}\right)$ with $T_{j} \stackrel{\text { def }}{=}$ 
$\int_{\mathscr{K}}^{\oplus} X_{j} \mathrm{~d} \mu_{n, m}\left(X_{1}, \ldots, X_{k}\right)$ and $T_{j}^{\prime} \stackrel{\text { def }}{=} \int_{\mathscr{K}}^{\oplus} X_{j} \mathrm{~d} \mu_{n, m^{\prime}}\left(X_{1}, \ldots, X_{k}\right)$. In other words (following [10]),

$$
\left(T_{1}, \ldots, T_{k}\right)=\int_{\mathscr{K}}^{\oplus} \text { id } \mathrm{d} \mu_{n, m} \quad \text { and } \quad\left(T_{1}^{\prime}, \ldots, T_{k}^{\prime}\right)=\int_{\mathscr{K}}^{\oplus} \text { id } \mathrm{d} \mu_{n, m^{\prime}}
$$

(where 'id' stands for the identity map). Since both $\mu_{n, m}$ and $\mu_{n, m^{\prime}}$ are regularity measures supported on, respectively, $\mathscr{S}(k) \cap \mathscr{M}_{n}^{k}$ and $\mathscr{S}(k) \cap \mathscr{M}_{n^{\prime}}^{k}$, it follows e.g. from Corollary 5.6.7 in [10] that $\mathcal{W}\left(T_{1}, \ldots, T_{k}\right)$ and $\mathcal{W}\left(T_{1}^{\prime}, \ldots, T_{k}^{\prime}\right)$ are, respectively, type $I_{n}$ and $I_{n^{\prime}}$ von Neumann algebras (because (2-4) are prime decompositions; consult [10] or Theorem 4.6 in Section 4 and the notes following it). This implies that $\left(T_{1}, \ldots, T_{k}\right) \perp_{u}\left(T_{1}^{\prime}, \ldots, T_{k}^{\prime}\right)$ and thus (2-3) holds, thanks to Lemma 5.4.10 in [10.

Having (2-3), one easily deduces from Lemma 5.6.12 (still in [10]) that $\mu$, which coincides with $\sum_{n=1}^{\infty} \sum_{m=1}^{\infty} \mu_{n, m}$, is a regularity measure.

\section{Prime Decomposition in Finite type I Algebras}

Let $A$ and $m$ be an $n \times n$ matrix and a positive integer, respectively. We denote by $m \odot A$ the inflation of $A$ composed of $m$ copies of $A$. That is,

$$
m \odot A=\left(\begin{array}{cccc}
A & 0 & \cdots & 0 \\
0 & A & \cdots & 0 \\
\vdots & \vdots & \ddots & \vdots \\
0 & 0 & \cdots & A
\end{array}\right)
$$

is a square matrix of degree $m n$. Additionally, for simplicity, we will write $\infty \odot A$ to denote the inflation of $A$ (acting on $\ell_{2}$ ) composed of countable infinite number of copies of $A$. In a similar manner we denote inflations of tuples of operators. We shall say a field $\left\{\left(X_{1}(\omega), \ldots, X_{k}(\omega)\right)\right\}_{\omega \in \Omega}$ of $k$-tuples is measurable if each of the fields $\left\{X_{s}(\omega)\right\}_{\omega \in \Omega}(s=1, \ldots, k)$ of operators is measurable (cf. [10]).

It is an easy exercise and a well-known fact that if $\{X(\omega)\}_{\omega \in \Omega}$ and $f: \Omega \rightarrow$ $\{1,2, \ldots, \infty\}$ are, respectively, a measurable field of matrices and a measurable function, then the field $\{f(\omega) \odot X(\omega)\}_{\omega \in \Omega}$ is measurable as well. What we want to prove is the following

3.1. Theorem. For $j \in\{1,2\}$, let $\left\{\left(X_{1}^{(j)}\left(\omega_{j}\right), \ldots, X_{k}^{(j)}\left(\omega_{j}\right)\right)\right\}_{\omega_{j} \in \Omega_{j}}$ and $f_{j}: \Omega_{j} \rightarrow$ $\{1,2, \ldots, \infty\}$ be, respectively, a measurable field of $k$-tuples of matrices and a measurable function defined on a standard measure space $\left(\Omega_{j}, \mathfrak{M}_{j}, \mu_{j}\right)$ such that:

(F1) for any $\omega_{j} \in \Omega_{j}$, the $k$-tuple $\left(X_{1}^{(j)}\left(\omega_{j}\right), \ldots, X_{k}^{(j)}\left(\omega_{j}\right)\right)$ is irreducible;

(F2) for any two distinct points $\omega_{j}$ and $\omega_{j}^{\prime}$ of $\Omega_{j}$, the $k$-tuples

$$
\left(X_{1}^{(j)}\left(\omega_{j}\right), \ldots, X_{k}^{(j)}\left(\omega_{j}\right)\right) \quad \text { and } \quad\left(X_{1}^{(j)}\left(\omega_{j}^{\prime}\right), \ldots, X_{k}^{(j)}\left(\omega_{j}^{\prime}\right)\right)
$$

are unitarily inequivalent.

Then the $k$-tuples

$$
\boldsymbol{T}_{1} \stackrel{\text { def }}{=}\left(\int_{\Omega_{1}}^{\oplus} f_{1}\left(\omega_{1}\right) \odot X_{1}^{(1)}\left(\omega_{1}\right) \mathrm{d} \mu_{1}\left(\omega_{1}\right), \ldots, \int_{\Omega_{1}}^{\oplus} f_{1}\left(\omega_{1}\right) \odot X_{k}^{(1)}\left(\omega_{1}\right) \mathrm{d} \mu_{1}\left(\omega_{1}\right)\right)
$$

and

$$
\boldsymbol{T}_{2} \stackrel{\text { def }}{=}\left(\int_{\Omega_{2}}^{\oplus} f_{2}\left(\omega_{2}\right) \odot X_{1}^{(2)}\left(\omega_{2}\right) \mathrm{d} \mu_{2}\left(\omega_{2}\right), \ldots, \int_{\Omega_{2}}^{\oplus} f_{2}\left(\omega_{2}\right) \odot X_{k}^{(2)}\left(\omega_{2}\right) \mathrm{d} \mu_{2}\left(\omega_{2}\right)\right)
$$

are unitarily equivalent iff there exist measurable sets $Z_{1} \subset \Omega_{1}, Z_{2} \subset \Omega_{2}$ and a Borel isomorphism $\Phi: \Omega_{1} \backslash Z_{1} \rightarrow \Omega_{2} \backslash Z_{2}$ such that:

(E1) $\mu_{1}\left(Z_{1}\right)=0$ and $\mu_{2}\left(Z_{2}\right)=0$; 
(E2) for each measurable set $B \subset \Omega_{1} \backslash Z_{1}, \mu_{2}(\Phi(B))=0$ if and only if $\mu_{1}(B)=0$;

(E3) the $k$-tuples $\left(X_{1}^{(2)}\left(\Phi\left(\omega_{1}\right)\right), \ldots, X_{k}^{(2)}\left(\Phi\left(\omega_{1}\right)\right)\right)$ and $\left(X_{1}^{(1)}\left(\omega_{1}\right), \ldots, X_{k}^{(1)}\left(\omega_{1}\right)\right)$ are unitarily equivalent and $f_{2}\left(\Phi\left(\omega_{1}\right)\right)=f_{1}\left(\omega_{1}\right)$ for any $\omega_{1} \in \Omega_{1} \backslash Z_{1}$.

Proof. The 'if' part is easy and well-known (and holds in more general settings; see e.g. items (di5)-(di6) on page 73 in [10]). Here we shall focus only on the 'only if' part. So, assume $\boldsymbol{T}_{1}$ and $\boldsymbol{T}_{2}$ are unitarily equivalent. Below we preserve the settings and notation of the previous section. Further, let $\mathscr{I}(k)$ denote $\bigcup_{n=1}^{\infty} \mathscr{I}_{n}(k)$ and $\psi: \mathscr{I}(k) \rightarrow \mathscr{S}(k)$ be a function given by the rule: $\psi\left(A_{1}, \ldots, A_{k}\right)$ is a unique $k$-tuple in $\mathscr{S}(k)$ which is unitarily equivalent to $\left(A_{1}, \ldots, A_{k}\right)$. Then $\psi$ is a Borel function. Now for $j \in\{1,2\}$, let $\varphi_{j}: \Omega_{j} \rightarrow \mathscr{S}(k)$ be given by $\varphi_{j}\left(\omega_{j}\right)=\psi\left(X_{1}^{(j)}\left(\omega_{j}\right), \ldots, X_{k}^{(j)}\left(\omega_{j}\right)\right)$. We see that $\varphi_{j}$ is measurable. What is more, it is one-to-one, by (F2). Since $\left(\Omega_{j}, \mathfrak{M}_{j}, \mu_{j}\right)$ is a standard Borel space, we conclude from Theorem 2.5 (involving e.g. the transport of $\mu_{j}$ under $\varphi_{j}$ ) that there is a measurable set $Z_{j}^{\prime} \subset \Omega_{j}$ such that $\mu_{j}\left(Z_{j}^{\prime}\right)=0$ and $\varphi_{j}\left(\Omega_{j} \backslash Z_{j}^{\prime}\right)$ is a measurable domain. This implies that the set $\left\{\left(X_{1}^{(j)}\left(\omega_{j}\right), \ldots, X_{k}^{(j)}\left(\omega_{j}\right)\right): \omega_{j} \in \Omega_{j} \backslash Z_{j}^{\prime}\right\}$ is a measurable domain as well. This means that the field $\left\{\left(X_{1}^{(j)}\left(\omega_{j}\right), \ldots, X_{k}^{(j)}\left(\omega_{j}\right)\right)\right\}_{\omega_{j} \in \Omega_{j}}$ is regular in the sense of [10] (cf. Proposition 5.4.4 there) and thus also

$$
\left\{\left(f_{j}\left(\omega_{j}\right) \odot X_{1}^{(j)}\left(\omega_{j}\right), \ldots, f_{j}\left(\omega_{j}\right) \odot X_{k}^{(j)}\left(\omega_{j}\right)\right)\right\}_{\omega_{j} \in \Omega_{j}} \text { is a regular field }
$$

(see Lemma 5.4.8 in [10]). To make further arguments more transparent, we need to recall some concepts introduced in $[10$. We consider the set $\mathfrak{F}$ of all unitary equivalence classes of (all) $k$-tuples of separable Hilbert space operators, equipped with a quotient Borel structure, as described in 10 . For simplicity, we denote by $\Phi_{j}: \Omega_{j} \rightarrow \mathfrak{F}$ a (measurable) function defined by the rule: $\Phi_{j}\left(\omega_{j}\right)$ is the unitary equivalence class of $\left(f_{j}\left(\omega_{j}\right) \odot X_{1}^{(j)}\left(\omega_{j}\right), \ldots, f_{j}\left(\omega_{j}\right) \odot X_{k}^{(j)}\left(\omega_{j}\right)\right)$. It follows from (3-1) and the choice of $Z_{j}^{\prime}$ that the set $\Phi_{j}\left(\Omega_{j} \backslash Z_{j}^{\prime}\right)$ is Borel in $\mathfrak{F}$ and the restriction of $\Phi_{j}$ to $\Omega_{j} \backslash Z_{j}^{\prime}$ is a Borel isomorphism; and from item (F2) that $\Phi_{j}$ is one-to-one (on the whole $\Omega_{j}$ ). Now let $\lambda_{j}$ be the transport of $\mu_{j}$ under $\Phi_{j}$; that is, $\lambda_{j}(\mathfrak{G})=$ $\mu_{j}\left(\Phi_{j}^{-1}(\mathfrak{G})\right)$ for any Borel set $\mathfrak{G} \subset \mathfrak{F}$. Since $\boldsymbol{T}_{1}$ and $\boldsymbol{T}_{2}$ are unitarily equivalent, we infer from Corollary 5.4.7 in [10] that the measures $\lambda_{1}$ and $\lambda_{2}$ are mutually absolutely continuous. So, the intersection $\mathfrak{F}_{0}$ of $\Phi_{1}\left(\Omega_{1} \backslash Z_{1}^{\prime}\right)$ and $\Phi_{2}\left(\Omega_{2} \backslash Z_{2}^{\prime}\right)$ is of full measure with respect to both $\lambda_{1}$ and $\lambda_{2}$. It suffices to put $Z_{j}=\Omega_{j} \backslash \Phi_{j}^{-1}\left(\mathfrak{F}_{0}\right)(\supset$ $Z_{j}^{\prime}$ ) and define $\Phi: \Omega_{1} \backslash Z_{1} \rightarrow \Omega_{2} \backslash Z_{2}$ as $\left(\left.\Phi_{2}\right|_{\Omega_{2} \backslash Z_{2}}\right)^{-1} \circ \Phi_{1}$. It is readily seen that $\Phi$ is a Borel isomorphism and conditions (E1)-(E2) are fulfilled. Finally, observe that for any $\omega_{1} \in \Omega_{1} \backslash Z_{1}, \Phi_{2}\left(\Phi\left(\omega_{1}\right)\right)=\Phi_{1}\left(\omega_{1}\right)$ which means that the $k$-tuples $\left(f_{2}\left(\Phi\left(\omega_{1}\right)\right) \odot X_{1}^{(2)}\left(\Phi\left(\omega_{1}\right)\right), \ldots, f_{2}\left(\Phi\left(\omega_{1}\right)\right) \odot X_{k}^{(2)}\left(\Phi\left(\omega_{1}\right)\right)\right)$ and $\left(f_{1}\left(\omega_{1}\right) \odot\right.$ $\left.X_{1}^{(1)}\left(\omega_{1}\right), \ldots, f_{1}\left(\omega_{1}\right) \odot X_{k}^{(1)}\left(\omega_{1}\right)\right)$ are unitarily equivalent. So, an application of (F1) yields that (E3) holds and we are done.

The proof of Theorem 1.1 depends on the property established below. The next result is almost certainly known, but we could not find it in the literature. (A similar, but slightly different, property was established by Ernest in Lemma 4.3 in [6.) For reader's convenience, we give its proof.

3.2. Lemma. If $\left\{\left(T_{1}(\omega), \ldots, T_{k}(\omega)\right)\right\}_{\omega \in \Omega}$ is a measurable field defined on a standard Borel space $(\Omega, \mathfrak{M})$ such that the $k$-tuples $\left(T_{1}(\omega), \ldots, T_{k}(\omega)\right)$ are unitarily equivalent to inflations of irreducible $k$-tuples of matrices, then there exist a measurable function $f: \Omega \rightarrow\{1,2, \ldots, \infty\}$ and a measurable field $\left\{\left(A_{1}(\omega), \ldots, A_{k}(\omega)\right)\right\}_{\omega \in \Omega}$ of irreducible $k$-tuples of matrices such that the $k$-tuples $\left(T_{1}(\omega), \ldots, T_{k}(\omega)\right)$ and $f(\omega) \odot\left(A_{1}(\omega), \ldots, A_{k}(\omega)\right)$ are unitarily equivalent for each $\omega \in \Omega$. 
Proof. The set $\Omega$ may be divided into countably many measurable parts, say $\Omega_{1}, \Omega_{2}, \ldots, \Omega_{\infty}$, such that $T_{s}(\omega)(s=1, \ldots, k)$ acts on a separable Hilbert space $\mathcal{H}_{n}$ of dimension $n$ for any $\omega \in \Omega_{n}$. Further, we may and do assume that $\mathcal{H}_{n}=\mathbb{C}^{n}$ for finite $n$ and $\mathcal{H}_{\infty}=\ell_{2}$. We now fix $n \in\{1,2, \ldots, \infty\}$ and put $J(n) \stackrel{\text { def }}{=}$ $\{p \in\{1,2, \ldots\}: n / p \in\{1,2, \ldots, \infty\}\}$. Everywhere below the algebra $\mathcal{B}\left(\mathcal{H}_{n}\right)$ of all bounded operators on $\mathcal{H}_{n}$ is equipped with the strong operator topology. Further, let $\mathscr{S}_{1}(k), \mathscr{S}_{2}(k), \ldots$ be sets obtained from Proposition 2.3. For any $p \in J(n)$ we use $\mathcal{U}_{p}$ to denote the set of all unitary operators on $\mathcal{H}_{n}$ which commute with any operator of the form $\frac{n}{p} \odot X_{s}$ for $s \in\{1, \ldots, k\}$ where $\left(X_{1}, \ldots, X_{k}\right) \in$ $\mathscr{S}_{p}(k)$. Then $\mathcal{U}_{p}$ is a closed subgroup of the unitary group of $\mathcal{H}_{n}$. By a theorem of Dixmier (see e.g. Theorem 12.17 in Chapter II of [7] or Theorem 1.2.4 in [1]), there exists a Borel set $\mathcal{E}_{p}$ of unitary operators on $\mathcal{H}_{n}$ which meets every left coset of $\mathcal{U}_{p}$ (in the whole unitary group) at exactly one point. Since $\mathscr{S}_{p}(k)$ consists of mutually unitarily inequivalent irreducible $k$-tuples, we conclude that the function $h_{n}: \bigcup_{p \in J(n)} \mathcal{E}_{p} \times \mathscr{S}_{p}(k) \rightarrow \mathcal{B}\left(\mathcal{H}_{n}\right)^{k}$ (where $\mathcal{B}\left(\mathcal{H}_{n}\right)$ is the algebra of all bounded operators on $\mathcal{H}_{n}$ equipped with the strong operator topology) given by $h_{n}\left(U,\left(A_{1}, \ldots, A_{k}\right)\right)=\left(U\left(\frac{n}{p} \odot A_{1}\right) U^{-1}, \ldots, U\left(\frac{n}{p} \odot A_{k}\right) U^{-1}\right)$ (for $\left.U \in \mathcal{E}_{p},\left(A_{1}, \ldots, A_{k}\right) \in \mathscr{S}_{p}(k)\right)$ is one-to-one. Furthermore, for any $\omega \in \Omega_{n}$ there is a unique pair $\left(U(\omega),\left(A_{1}(\omega), \ldots, A_{k}(\omega)\right)\right) \in \bigcup_{p \in J(n)} \mathcal{E}_{p} \times \mathscr{S}_{p}(k)$ for which $\left(T_{1}(\omega), \ldots, T_{k}(\omega)\right)=h_{n}\left(U(\omega),\left(A_{1}(\omega), \ldots, A_{k}(\omega)\right)\right)$. Since $h_{n}$ is one-to-one, it is a Borel isomorphisms and thus the field $\left\{\left(A_{1}(\omega), \ldots, A_{k}(\omega)\right)\right\}_{\omega \in \Omega_{n}}$ is measurable. Now it suffices to put $f(\omega) \stackrel{\text { def }}{=} \frac{n}{p}$ provided $\omega \in \Omega_{n}$ is such that $\left(A_{1}(\omega), \ldots, A_{k}(\omega)\right) \in$ $\mathscr{S}_{p}(k)$. The very definition of $f$ shows that $f$ is measurable and the whole construction yields the assertion of the lemma.

Below we give a proof of Theorem 1.1 in more general settings; that is, for $k$-tuples in place of single operators.

Proof of Theorem 1.1. Assume all the assumptions of item (a) are fulfilled. Then it follows from Lemma 3.2 that we may find measurable fields $\left\{\left(A_{j}\left(\omega_{j}\right)\right\}_{\omega_{j} \in \Omega_{j}}(j=\right.$ $1,2)$ of irreducible matrices and measurable functions $f_{j}: \Omega_{j} \rightarrow\{1,2, \ldots, \infty\}(j=$ $1,2)$ such that $T_{j}\left(\omega_{j}\right)$ is unitarily equivalent to $f_{j}\left(\omega_{j}\right) \odot A_{j}\left(\omega_{j}\right)$ for $\mu_{j}$-almost all $\omega_{j} \in \Omega_{j}$. We conclude that then $\int_{\Omega_{1}}^{\oplus} f_{1}\left(\omega_{1}\right) \odot A_{1}\left(\omega_{1}\right) \mathrm{d} \mu_{1}\left(\omega_{1}\right)$ is unitarily equivalent to $\int_{\Omega_{2}}^{\oplus} f_{2}\left(\omega_{2}\right) \odot A_{2}\left(\omega_{2}\right) \mathrm{d} \mu_{2}\left(\omega_{2}\right)$. Now an application of Theorem 3.1 easily finishes the proof of that part.

We turn to (b). First of all, observe that $T$ is affiliated with a finite type I von Neumann algebra iff $\mathcal{W}^{\prime \prime}(T)$ is such an algebra (because $T$ is affiliated with $\mathcal{M}$ iff $\mathcal{M}^{\prime} \subset \mathcal{W}^{\prime}(T)$, iff $\mathcal{W}^{\prime \prime}(T) \subset \mathcal{M}$; and being finite and type I is a hereditary property for von Neumann algebras). So, if $\mathcal{W}^{\prime \prime}(T)$ is finite and type I, the conclusion follows from Ernest's central decomposition [6] or our Prime Decomposition Theorem [10]. Conversely, if $T$ is unitarily equivalent to (1-3) where $\{T(\omega)\}$ and $(\Omega, \mathfrak{M}, \mu)$ are as in item (b) of the theorem, then we argue as follows. Lemma 3.2 shows we may replace $T(\omega)$ by $f(\omega) \odot A(\omega)$ where $A(\omega)$ 's are mutually unitarily inequivalent irreducible matrices. Further, the proof of Theorem 3.1 includes the information that then the field $\{f(\omega) \odot A(\omega)\}_{\omega \in \Omega}$ is regular and hence $\int_{\Omega}^{\oplus} f(\omega) \odot A(\omega) \mathrm{d} \mu(\omega)$ is the prime decomposition of $T$ (for details, consult [10]). Hence $\mathcal{W}^{\prime \prime}(T)$ is finite and type I, because $\mathcal{W}^{\prime \prime}(A(\omega))$ is so for any $\omega \in \Omega$ (see Corollary 5.6.7 in [10]).

3.3. Remark. The reasoning presented above proves also a counterpart of Theorem 1.1 for $k$-tuples of operators, whose precise formulation and detailed proof are left to the reader as an exercise. 


\section{Finite TyPE I IDEALS}

First we recall the concept of an ideal introduced in [10].

4.1. Definition. A class $\mathcal{J}$ of $k$-tuples of operators is said to be an ideal if $\mathcal{J}$ is nonempty and each of the following three conditions is fulfilled:

(ID0) if $\left(T_{1}, \ldots, T_{k}\right) \in \mathcal{J}$ and a $k$-tuple $\left(T_{1}^{\prime}, \ldots, T_{k}^{\prime}\right)$ (acting in a totally arbitrary Hilbert space) is unitarily equivalent to $\left(T_{1}, \ldots, T_{k}\right)$, then $\left(T_{1}^{\prime}, \ldots, T_{k}^{\prime}\right) \in \mathcal{J}$;

(ID1) if $\left\{\left(T_{1}^{(s)}, \ldots, T_{k}^{(s)}\right)\right\}_{s \in S}$ is a nonempty collection of $k$-tuples belonging to J , then $\left(\bigoplus_{s \in S} T_{1}^{(s)}, \ldots, \bigoplus_{s \in S} T_{k}^{(s)}\right) \in \mathcal{J}$;

(ID2) whenever $\left(T_{1}, \ldots, T_{k}\right)$ is a member of $\mathcal{J}$ acting in a Hilbert space $\mathcal{H}$ and $\mathcal{E}$ is a closed linear subspace of $\mathcal{H}$ (including trivial) which reduces each of $T_{1}, \ldots, T_{k}$, then $\left(\left.T_{1}\right|_{\mathcal{E}}, \ldots,\left.T_{k}\right|_{\mathcal{E}}\right)$ belongs to $\mathcal{J}$ as well.

Ideals are natural generalisations (to $k$-tuples of operators) of part-properties studied by Brown, Fong and Hadwin in [2].

For simplicity, we extend the notation (for inflations) introduced in the previous section to arbitrary cardinal numbers: whenever $\alpha$ is a positive cardinal number and $\left(X_{1}, \ldots, X_{k}\right)$ is a $k$-tuple of operators acting in a Hilbert space $\mathcal{H}$, then $\alpha \odot\left(X_{1}, \ldots, X_{k}\right)$ denotes the inflation of $\left(X_{1}, \ldots, X_{k}\right)$ consisting of $\alpha$ copies of this tuple; more formally, $\alpha \odot\left(X_{1}, \ldots, X_{k}\right)$ acts in $\bigoplus_{\xi<\bar{\alpha}} \mathcal{H}$ and coincides with $\left(\bigoplus_{\xi<\bar{\alpha}} X_{1}, \ldots, \bigoplus_{\xi<\bar{\alpha}} X_{k}\right)$ where $\bar{\alpha}$ is the initial ordinal of cardinality $\alpha$.

In the sequel we shall need the following characterisation of ideals, formulated as Corollary 3.6.6 in [10].

4.2. Lemma. A nonempty class $\mathcal{J}$ of $k$-tuples of operators which satisfies condition (ID0) is an ideal iff the following two conditions are fulfilled:

(ID1') whenever $\left(T_{1}^{(s)}, \ldots, T_{k}^{(s)}\right)_{s \in S} \subset \mathcal{J}$ is a nonempty collection of mutually unitarily disjoint $k$-tuples of operators acting in separable Hilbert spaces, then $\left(\bigoplus_{s \in S} T_{1}^{(s)}, \ldots, \bigoplus_{s \in S} T_{k}^{(s)}\right) \in \mathcal{J}$

(ID2') if $\left(X_{1}, \ldots, X_{k}\right)$ and $\left(Y_{1}, \ldots, Y_{k}\right)$ are two unitarily disjoint $k$-tuples such that $\left(X_{1} \oplus Y_{1}, \ldots, X_{k} \oplus Y_{k}\right) \in \mathcal{J}$, then $\left(X_{1}, \ldots, X_{k}\right) \in \mathcal{J}$;

(ID3') for any $k$-tuple $\left(X_{1}, \ldots, X_{k}\right)$ and each positive cardinal number $\alpha, \alpha \odot$ $\left(X_{1}, \ldots, X_{k}\right) \in \mathcal{J}$ iff $\left(X_{1}, \ldots, X_{k}\right) \in \mathcal{J}$.

4.3. Lemma. Let $\mathrm{J}_{I}^{f}$ be the class of all $k$-tuples $\left(T_{1}, \ldots, T_{k}\right)$ of operators (including all those acting in zero-dimensional Hilbert spaces) such that $\mathcal{W}^{\prime \prime}\left(T_{1}, \ldots, T_{k}\right)$ is finite and type I. Then $\mathcal{J}_{I}^{f}$ is an ideal.

Proof. Let $\mathcal{J}_{I}$ and $\mathcal{J}^{f}$ be the classes of all $k$-tuples $\left(T_{1}, \ldots, T_{k}\right)$ (including all those acting in zero-dimensional Hilbert spaces) such that $\mathcal{W}^{\prime \prime}\left(T_{1}, \ldots, T_{k}\right)$ is type $\mathrm{I}$ and, respectively, finite. Then $\mathcal{J}_{I}^{f}=\mathcal{J}^{f} \cap \mathcal{J}_{I}$, and $\mathcal{J}_{I}$ is an ideal, by [10. Therefore it is enough to show that $\mathcal{J}^{f}$ is an ideal. It is clear that (ID0) is satisfied. If $\left\{\left(T_{1}^{(s)}, \ldots, T_{k}^{(s)}\right)\right\}_{s \in S}$ is a nonempty collection of members of $\mathcal{J}^{f}$, then the algebra $\mathcal{W}^{\prime \prime}\left(\bigoplus_{s \in S} T_{1}^{(s)}, \ldots, \bigoplus_{s \in S} T_{k}^{(s)}\right)$ is (naturally) *-isomorphic to a unital subalgebra of $\prod_{s \in S} \mathcal{W}^{\prime \prime}\left(T_{1}^{(s)}, \ldots, T_{k}^{(s)}\right)$ and thus $\mathcal{W}^{\prime \prime}\left(\bigoplus_{s \in S} T_{1}^{(s)}, \ldots, \bigoplus_{s \in S} T_{k}^{(s)}\right)$ is finite. This shows (ID1) as well as (ID1'). Further, since $\mathcal{W}^{\prime \prime}\left(\alpha \odot\left(X_{1}, \ldots, X_{k}\right)\right)$ is *isomorphic to $\mathcal{W}^{\prime \prime}\left(X_{1}, \ldots, X_{k}\right)$, we see that also (ID3') holds. So, it remains to check (ID2'). To this end, assume $\left(X_{1}, \ldots, X_{k}\right)$ and $\left(Y_{1}, \ldots, Y_{k}\right)$ are two unitarily disjoint $k$-tuples such that $\left(X_{1} \oplus Y_{1}, \ldots, X_{k} \oplus Y_{k}\right) \in \mathcal{J}^{f}$. We infer from the unitary disjointness of them that $\mathcal{W}^{\prime \prime}\left(X_{1} \oplus Y_{1}, \ldots, X_{k} \oplus Y_{k}\right)$ is *-isomorphic to $\mathcal{W}^{\prime \prime}\left(X_{1}, \ldots, X_{k}\right) \times \mathcal{W}^{\prime \prime}\left(Y_{1}, \ldots, Y_{k}\right)$ which implies that both the von Neumann algebras $\mathcal{W}^{\prime \prime}\left(X_{1}, \ldots, X_{k}\right)$ and $\mathcal{W}^{\prime \prime}\left(Y_{1}, \ldots, Y_{k}\right)$ are finite. This completes the proof. 
We now introduce

4.4. Definition. An ideal $\mathcal{J}$ of $k$-tuples is said to be finite and type $I$ if $\mathcal{J} \subset \mathcal{J}_{I}^{f}$.

To every ideal of $k$-tuples there naturally corresponds an ideal of unitary equivalence classes of $k$-tuples (based on (ID0)). We shall identify both these concepts (i.e., in the realms of 'concrete' $k$-tuples and their unitary equivalence classes). In [10 we formulated Prime Decomposition Theorem for totally arbitrary ideals of unitary equivalence classes of $k$-tuples. It involved so-called coverings defined on (so-called) multi-standard measurable spaces with nullities which we now recall. A measurable space with nullity is a triple $(\mathscr{X}, \mathfrak{M}, \mathcal{N})$ where $\mathscr{X}$ is a set, $\mathfrak{M}$ a $\sigma$-algebra of subsets of $\mathscr{X}$ and $\mathcal{N}$ is a $\sigma$-ideal in $\mathfrak{M}$. $\mathcal{N}$ is a counterpart of the $\sigma$-ideal of all sets of measure zero in a measure space and thus its members are called null sets. A set $\mathscr{B} \in \mathfrak{M}$ in a measurable space with nullity $(\mathscr{X}, \mathfrak{M}, \mathcal{N})$ is called standard if there exists a $\sigma$-finite (or, equivalently, finite) positive measure $\mu$ on $\left.\mathfrak{M}\right|_{\mathscr{B}} \stackrel{\text { def }}{=}\{\mathscr{A} \in \mathfrak{M}: \mathscr{A} \subset \mathscr{B}\}$ such that the $\sigma$-ideals $\mathcal{N}(\mu) \stackrel{\text { def }}{=}\left\{\left.\mathscr{Z} \in \mathfrak{M}\right|_{\mathscr{B}}: \mu(\mathscr{Z})=0\right\}$ and $\left.\mathcal{N}\right|_{\mathscr{B}} \stackrel{\text { def }}{=}\{\mathscr{Z} \in \mathcal{N}: \quad \mathscr{Z} \subset \mathscr{B}\}$ coincide, and $\left(\mathscr{B},\left.\mathfrak{M}\right|_{\mathscr{B}}, \mu\right)$ is a standard measure space. Finally, $(\mathscr{X}, \mathfrak{M}, \mathcal{N})$ is multi-standard if there exists a collection (called a standard base) $\left\{\mathscr{B}_{s}\right\}_{s \in S} \subset \mathfrak{M}$ of pairwise disjoint sets satisfying the following conditions:

- $\mathscr{B}_{s}$ is standard for any $s \in S$;

- $\mathscr{X} \backslash \bigcup_{s \in S} \mathscr{B}_{s} \in \mathcal{N}$;

- a subset $\mathscr{A}$ of $\bigcup_{s \in S} \mathscr{B}_{s}$ belongs to $\mathfrak{M}$ iff $\mathscr{A} \cap \mathscr{B}_{s} \in \mathfrak{M}$ for each $s \in S$;

- an arbitrary set $\mathscr{A} \subset \bigcup_{s \in S} \mathscr{B}_{s}$ belongs to $\mathcal{N}$ iff $\mathscr{A} \cap \mathscr{B}_{s} \in \mathcal{N}$ for all $s \in S$.

Next, we recall so-called 'continuous' direct sums of measurable fields defined on multi-standard measurable spaces with nullities. To make the presentation shorter, here we restrict only to measurable fields whose values are irreducible $k$-tuples of matrices. To this end, let $\mathfrak{I}_{n}(k)$ be as in Definition[2.2 and let $\mathfrak{I}(k)=\bigsqcup_{n=1}^{\infty} \mathfrak{I}_{n}(k)$ be the topological disjoint union of all $\mathfrak{I}_{n}(k)$ 's (with fixed $k$ ). Then $\mathfrak{I}(k)$ is separable, locally compact and metrizable. Further, let $\mathscr{S}(k)$ be as in Section 2 (that is, $\mathscr{S}(k)$ meets the unitary equivalence class of each irreducible $k$-tuple of matrices at exactly one point). Now let $(\mathscr{X}, \mathfrak{M}, \mathcal{N})$ be a multi-standard measurable space with nullity, $\left\{\mathscr{B}_{s}\right\}_{s \in S}$ be its standard base and for each $s \in S$, let $\mu_{s}$ be a standard measure on $\left.\mathfrak{M}\right|_{\mathscr{B}_{s}}$ such that $\mathcal{N}\left(\mu_{s}\right)=\left.\mathcal{N}\right|_{\mathscr{B}_{s}}$. A cardinal-valued function $f: \mathscr{X} \rightarrow$ Card is said to be measurable if:

- $f^{-1}(\{\alpha\}) \in \mathfrak{M}$ for any cardinal $\alpha$;

- for any standard set $\mathscr{B} \in \mathfrak{M}$ there is $\mathscr{Z} \in \mathcal{N}$ such that the set $f(\mathscr{B} \backslash \mathscr{Z})$ is countable (finite or not).

Additionally, $f$ is nontrivial if $\mathscr{X} \backslash f^{-1}(\{0\}) \notin \mathcal{N}$. For a measurable function $\Phi: \mathscr{X} \rightarrow \mathfrak{I}(k)$ and a measurable nontrivial cardinal-valued function $f: \mathscr{X} \rightarrow$ Card one defines (following [10]) $\bigoplus_{x \in \mathscr{X}}^{\mathcal{N}} f(x) \odot \Phi(x)$ as the unitary equivalence class of the $k$-tuple $\left(X_{1}, \ldots, X_{k}\right)$ where

$$
X_{j}=\bigoplus_{\alpha>0} \alpha \odot\left(\bigoplus_{s \in S} \int_{\mathscr{B}_{s, \alpha}}^{\oplus} T_{j}(x) \mathrm{d} \mu_{s}(x)\right)
$$

with $\mathscr{B}_{s, \alpha}=\mathscr{B}_{s} \cap f^{-1}(\{\alpha\})$ and for each $x \in \mathscr{X},\left(T_{1}(x), \ldots, T_{k}(x)\right)$ is a (unique) member of $\mathscr{S}(k)$ whose unitary equivalence class coincides with $\Phi(x)$. One proves (consult [10]) that the above definition is correct; that is, it is independent of the choice of the transversal $\mathscr{S}(k)$, standard base $\left\{\mathscr{B}_{s}\right\}_{s \in S}$ and standard measures $\mu_{s}$ 's.

Finally, a covering is any measurable field $\Phi: \mathscr{X} \rightarrow \mathfrak{I}(k)$ defined on a multistandard measurable space with nullity $(\mathscr{X}, \mathfrak{M}, \mathcal{N})$ such that $\bigoplus_{x \in \mathscr{B}}^{\mathcal{N}} \Phi(x)$ is unitarily disjoint from $\bigoplus_{x \in \mathscr{B}^{\prime}}^{\mathcal{N}} \Phi(x)$ for any two disjoint sets $\mathscr{B}, \mathscr{B}^{\prime} \in \mathfrak{M}$. Coverings were 
characterised in Theorem 5.5.5 in [10. Below we formulate its equivalent form for coverings with values in $\mathfrak{I}(k)$. Below $\psi: \mathfrak{I}(k) \rightarrow \mathscr{S}(k)$ is a function given by the rule: for each $\mathfrak{D} \in \mathfrak{I}(k), \psi(\mathfrak{D})$ is a unique point of $\mathfrak{D} \cap \mathscr{S}(k)$.

4.5. Theorem. A measurable function $\Phi: \mathscr{X} \rightarrow \mathfrak{I}(k)$ defined on a multi-standard measurable space with nullity $(\mathscr{X}, \mathfrak{M}, \mathcal{N})$ is a covering iff for any standard set $\mathscr{B} \in$ $\mathfrak{M}$ there is $\mathscr{Z} \in \mathcal{N}$ such that $\psi(\Phi(\mathscr{B} \backslash \mathscr{Z}))$ is a measurable domain and the restriction of $\psi \circ \Phi$ to $\mathscr{B} \backslash \mathscr{Z}$ is a Borel isomorphism (of $\mathscr{B} \backslash \mathscr{Z}$ onto its image).

Prime Decomposition Theorem (Theorem 5.6.14 in [10]) for finite type I ideals reads as follows.

4.6. Theorem. Let $\mathcal{J}$ be a finite type I ideal of unitary equivalence classes of $k$ tuples.

(A) There exists a covering $\Phi: \mathscr{X} \rightarrow \mathfrak{I}(k)$ defined on $(\mathscr{X}, \mathfrak{M}, \mathcal{N})$ such that

$$
\mathcal{J}=\mathcal{J}(\Phi) \stackrel{\text { def }}{=}\left\{\bigoplus_{x \in \mathscr{X}}^{\mathcal{N}} f(x) \odot \Phi(x) \mid \quad f: \mathscr{X} \rightarrow \text { Card measurable }\right\} .
$$

(B) If $\Phi: \mathscr{X} \rightarrow \mathfrak{I}(k)$ and $\Phi: \mathscr{X}^{\prime} \rightarrow \mathfrak{I}(k)$ are coverings defined on, respectively, $(\mathscr{X}, \mathfrak{M}, \mathcal{N})$ and $\left(\mathscr{X}^{\prime}, \mathfrak{M}^{\prime}, \mathcal{N}^{\prime}\right)$ such that $\mathcal{J}(\Phi)=\mathcal{J}\left(\Phi^{\prime}\right)$, then there are sets $\mathscr{Z} \in$ $\mathcal{N}, \mathscr{Z}^{\prime} \in \mathcal{N}^{\prime}$ and a Borel isomorphism $\Psi: \mathscr{X} \backslash \mathscr{Z} \rightarrow \mathscr{X}^{\prime} \backslash \mathscr{Z}^{\prime}$ such that $\left\{\Psi(\mathscr{B}):\left.\mathscr{B} \in \mathcal{N}\right|_{\mathscr{Z}}\right\}=\left.\mathcal{N}^{\prime}\right|_{\mathscr{Z}}$, and $\Phi^{\prime}(\Psi(x))=\Phi^{\prime}(x)$ for any $x \in \mathscr{X} \backslash \mathscr{X}^{\prime}$.

(C) If $\Phi: \mathscr{X} \rightarrow \mathfrak{I}(k)$ is a covering as in $(\mathrm{A})$, then for two measurable functions $f, g: \mathscr{X} \rightarrow$ Card one has:

- $\bigoplus_{x \in \mathscr{X}}^{\mathcal{N}} f(x) \odot \Phi(x)=\bigoplus_{x \in \mathscr{X}}^{\mathcal{N}} g(x) \odot \Phi(x)$ iff $f=g$ a.e.; that is, iff $\{x \in \mathscr{X}: f(x) \neq g(x)\} \in \mathcal{N}$;

- $\bigoplus_{x \in \mathscr{X}}^{\mathcal{N}}(f(x)+g(x)) \odot \Phi(x)=\left(\bigoplus_{x \in \mathscr{X}}^{\mathcal{N}} f(x) \odot \Phi(x)\right) \oplus\left(\bigoplus_{x \in \mathscr{X}}^{\mathcal{N}} g(x) \odot \Phi(x)\right)$. (D) If $\Phi: \mathscr{X} \rightarrow \mathfrak{I}(k)$ is a covering, then $\mathcal{J}(\Phi)$ is a finite type I ideal.

The above result establishes a one-to-one correspondence between finite type I ideals (of unitary equivalence classes of $k$-tuples) and, up to isomorphism (in the sense of item (B) above), coverings taking values in $\mathfrak{I}(k)$. Furthermore, if $\mathcal{J}$ is any such ideal and $\Phi: \mathscr{X} \rightarrow \mathfrak{I}(k)$ is its covering on $(\mathscr{X}, \mathfrak{M}, \mathcal{N})$, then for every $\mathrm{X} \in \mathcal{J}$ there exists a unique (up to $\mathcal{N}$-almost everywhere equality) cardinal-valued measurable function $m_{\mathrm{X}}: \mathscr{X} \rightarrow$ Card such that

$$
\mathrm{X}=\bigoplus_{x \in \mathscr{X}}^{\mathcal{N}} m_{\mathrm{X}}(x) \odot \Phi(x)
$$

The above representation is called the prime decomposition of $\mathbf{X}$ and $m_{\mathbf{X}}$ is said to be the multiplicity function of $\mathrm{X}$ (both in $\mathcal{J}$ ), see 10 .

So, any characterisation of coverings helps understanding the complexity of ideals. Below we give a simple criterion for an $\mathfrak{I}(k)$-valued function to be a covering.

4.7. Proposition. A measurable function $\Phi: \mathscr{X} \rightarrow \mathfrak{I}(k)$ defined on $(\mathscr{X}, \mathfrak{M}, \mathcal{N})$ is a covering iff for every standard set $\mathscr{B} \in \mathfrak{M}$ there is $\mathscr{Z} \in \mathcal{N}$ such that $\Phi$ is one-to-one on $\mathscr{B} \backslash \mathscr{Z}$.

Proof. We only need to show the 'if' part of the proposition. Assume $\mathscr{B}$ is a standard set such that the restriction of $\Phi$ to $\mathscr{B}$ is one-to-one. We may and do assume that $\left(\mathscr{B},\left.\mathfrak{M}\right|_{\mathscr{B}}\right)$ is a standard Borel space. Let $\mu$ be a finite measure on $\left.\mathfrak{M}\right|_{\mathscr{B}}$ for which $\mathcal{N}(\mu)=\left.\mathcal{N}\right|_{\mathscr{B}}$. Since $\mathscr{B}$ and $\mathfrak{I}(k)$ are standard Borel spaces, we conclude that $\Phi(\mathscr{B})$ is Borel and the restriction of $\Phi$ to $\mathscr{B}$ is a Borel isomorphism. Let $\psi: \mathfrak{I}(k) \rightarrow \mathscr{S}(k)$ be as in Theorem 4.5. Denote by $\lambda$ the transport of $\mu$ under $\psi \circ \Phi$ (that is, $\lambda(\mathscr{A})=\mu\left(\Phi^{-1}\left(\psi^{-1}(\mathscr{A})\right)\right)$ for any $\left.\mathscr{A} \in \mathfrak{B}(\mathscr{S}(k))\right)$. We infer from Theorem 2.5 that there is a measurable domain $\mathscr{F} \subset \mathscr{S}(k)$ such that $\lambda(\mathscr{S}(k) \backslash \mathscr{F})=$ 
0. Now we put $\mathscr{Z} \stackrel{\text { def }}{=} \mathscr{B} \backslash \Phi^{-1}\left(\psi^{-1}(\mathscr{F})\right) \in \mathfrak{M}$. Observe that $\mu(\mathscr{Z})=0$ and hence $\mathscr{Z} \in \mathcal{N}$. What is more, $\psi(\Phi(\mathscr{B} \backslash \mathscr{Z})) \subset \mathscr{F}$ and thus $\psi(\Phi(\mathscr{B} \backslash \mathscr{Z}))$ is a measurable domain. So, an application of Theorem 4.5 finishes the proof.

With the help of Proposition 4.7 we can now describe a covering for $\mathcal{J}_{I}^{f}$.

4.8. Proposition. Let $\mathscr{M}=\left\{\mu_{s}\right\}_{s \in S}$ be a maximal collection of mutually singular probabilistic Borel measures on $\mathfrak{I}(k)$. Put $\mathscr{X} \stackrel{\text { def }}{=} \mathfrak{I}(k) \times S$,

$$
\begin{gathered}
\mathfrak{M} \stackrel{\text { def }}{=}\left\{\mathscr{B} \subset \mathscr{X} \mid \quad \forall s \in S: \mathscr{B}^{s} \in \mathfrak{B}(\mathfrak{I}(k))\right\}, \\
\mathcal{N} \stackrel{\text { def }}{=}\left\{\mathscr{Z} \in \mathfrak{M} \mid \quad \forall s \in S: \mathscr{B}^{s} \in \mathcal{N}\left(\mu_{s}\right)\right\}
\end{gathered}
$$

where $\mathscr{B}^{s}=\{\mathrm{X} \in \mathfrak{I}(k):(\mathrm{X}, s) \in \mathscr{B}\}$ for each $s \in S$; and let $\Phi: \mathscr{X} \rightarrow \mathfrak{I}(k)$ be the projection onto the first coordinate. Then $(\mathscr{X}, \mathfrak{M}, \mathcal{N})$ is a multi-standard measurable space with nullity and $\Phi$ is a covering such that $\mathcal{J}(\Phi)=\mathcal{J}_{I}^{f}$.

Proof. It is clear that $(\mathscr{X}, \mathfrak{M}, \mathcal{N})$ is multi-standard and $\{\mathfrak{I}(k) \times\{s\}\}_{s \in S}$ is its standard base. There is also no difficulty in checking that $\Phi$ is measurable. We shall now show that $\Phi$ is a covering. Let $\mathscr{B} \in \mathfrak{M}$ be a standard set in $\mathscr{X}$. According to Proposition 4.7 it suffices to show that there is $\mathscr{Z} \in \mathcal{N}$ such that the restriction of $\Phi$ to $\mathscr{B} \backslash \mathscr{Z}$ is one-to-one. To this end, first observe that are only countably many indices $s \in S$ for which $\mathscr{B}^{s} \notin \mathcal{N}\left(\mu_{s}\right)$. Denote by $S_{0}$ the set of all such $s \in S$. Since the measures $\mu_{s}\left(s \in S_{0}\right)$ are mutually singular, we conclude from the countability of $S_{0}$ that there is a collection $\left\{\mathfrak{D}_{s}\right\}_{s \in S_{0}}$ of pairwise disjoint Borel subsets of $\mathfrak{I}(k)$ such that $\mu_{s}\left(\mathfrak{D}_{s}\right)=1$ for each $s \in S_{0}$. Then $\mathscr{Z} \stackrel{\text { def }}{=} \mathscr{B} \backslash \bigcup_{s \in S_{0}}\left(\mathfrak{D}_{s} \times\{s\}\right)$ is a member of $\mathcal{N}$ we searched for.

It remains to check that $\mathcal{J}(\Phi)=\mathcal{J}_{I}^{f}$. Inclusion ' $C$ ' follows from item (D) of Theorem 4.6. The reverse inclusion may simply be deduced from the proof of Proposition 5.6.13 in [10]. Below we repeat its main arguments. Denote by $\mathrm{J}_{I}^{f}$ the so-called unit of $\mathcal{J}_{I}^{f}$ (to read more on unities of ideals, consult $\S 3.5$ in [10]) and put $\mathrm{T} \stackrel{\text { def }}{=} \bigoplus_{x \in \mathscr{X}}^{\mathcal{N}} \Phi(x)$. Then there exists a $k$-tuple of operators whose unitary equivalence class $\mathrm{X}$ satisfies: $\mathrm{J}_{I}^{f}=\mathrm{T} \oplus \mathrm{X}$ and $\mathrm{T} \perp_{u} \mathrm{X}$. As in the proof mentioned above, it suffices to show that $X$ is trivial (i.e. its representatives act on zerodimensional Hilbert spaces). Assuming, on the contrary, that $\mathbf{X}$ is nontrivial, we conclude that there is a Borel probabilistic measure $\mu$ on $\mathcal{J}(k)$ such that $\mathrm{X}_{0} \perp_{u} \mathrm{~T}$ and $\mathrm{X}_{0} \in \mathcal{J}_{I}^{f}$ where $\mathbf{X}_{0}=\bigoplus_{\mathrm{T} \in \mathfrak{I}(k)}^{\mathcal{N}(\mu)} \mathrm{T}$. (The fact that $\mu$ is defined on $\mathfrak{I}(k)$ follows from the latter property of $\mathrm{X}_{0}$.) Now the unitary disjointness of $\mathrm{X}_{0}$ and $\mathrm{T}$ implies that $\mu$ is singular to each of the measures $\mu_{s}(s \in S)$, which contradicts the maximality of the collection $\mathscr{M}$.

As a consequence of Proposition 4.8 and Theorem 4.6 we obtain

4.9. Corollary. Let $\mathscr{M},(\mathscr{X}, \mathfrak{M}, \mathcal{N})$ and $\Phi$ be as in Proposition 4.8. For every $\mathrm{X} \in \mathcal{J}_{I}^{f}$ there exists a (unique up to $\mathcal{N}$-almost everywhere equality) measurable cardinal-valued function $m_{\mathrm{X}}: \mathscr{X} \rightarrow$ Card such that $\mathrm{X}=\bigoplus_{x \in \mathscr{X}}^{\mathcal{N}} m_{\mathrm{X}}(x) \odot \Phi(x)$.

We are now ready to turn to the main subject of the section.

4.10. Definition. Let $\mathscr{M},(\mathscr{X}, \mathfrak{M}, \mathcal{N})$ and $\Phi$ be as above. For any Borel subset $\mathfrak{F}$ of $\mathfrak{I}(k)$ let $\mathcal{J}[\mathfrak{F}]$ be the class of all unitary equivalence classes $\mathbf{X}$ for which $m_{\mathbf{X}}(x)=0$ for $\mathcal{N}$-almost all $x \in \Phi^{-1}(\mathfrak{F})$.

Since coverings are unique (up to isomorphism), the definition of $\mathcal{J}[\mathfrak{F}]$ introduced below is independent of the choice of the collection $\mathscr{M}$ of mutually singular measures.

Our first claim is 
4.11. Lemma. For each Borel set $\mathfrak{F} \subset \mathfrak{I}(k)$, the class $\mathfrak{J}[\mathfrak{F}]$ is an ideal of unitary equivalence classes of $k$-tuples.

Proof. Let $\mathscr{M},(\mathscr{X}, \mathfrak{M}, \mathcal{N})$ and $\Phi$ be as usual. Let $j_{\mathfrak{F}}: \mathscr{X} \rightarrow\{0,1\}(\subset$ Card $)$ be the characteristic function of $\Phi^{-1}(\mathfrak{F})$ and put $\mathrm{J}[\mathfrak{F}] \stackrel{\text { def }}{=} \bigoplus_{x \in \mathscr{X}}^{\mathcal{N}} j_{\mathfrak{F}}(x) \odot \Phi(x)$. Then for an arbitrary measurable function $m: \mathscr{X} \rightarrow$ Card we have:

$$
\bigoplus_{x \in \mathscr{X}}^{\mathcal{N}} m(x) \odot \Phi(x) \in \mathcal{J}[\mathfrak{F}] \Longleftrightarrow m(x) \cdot j_{\mathfrak{F}}(x)=m(x) \text { for } \mathcal{N} \text {-almost all } x \in \mathscr{X} \text {. }
$$

According to Theorem 5.6.14 in [10], the above is equivalent to:

$$
\mathrm{X} \in \mathrm{J}[\mathfrak{F}] \Longleftrightarrow \mathrm{X} \ll \mathrm{J}[\mathfrak{F}]
$$

(where $\mathrm{A} \ll \mathrm{B}$ means that $\alpha \odot \mathrm{B}=\mathrm{A} \oplus \mathrm{S}$ for some cardinal $\alpha$ and a unitary equivalence class $S)$. Finally, again from [10] it follows that the class $\{X: X \ll J[\mathfrak{F}]\}$ is an ideal.

Mimicing the proof of Proposition 4.7, one shows that

4.12. Proposition. Let $\mathfrak{F}$ be a Borel subset of $\mathfrak{I}(k)$ and let $\mathscr{M}_{\mathfrak{F}}=\left\{\mu_{s}\right\}_{s \in S}$ be a maximal collection of mutually singular probabilistic Borel measures on $\mathfrak{I}(k)$ each of which is supported on $\mathfrak{F}$. Put $\mathscr{X}_{\mathfrak{F}} \stackrel{\text { def }}{=} \mathfrak{F} \times S$,

$$
\begin{gathered}
\mathfrak{M}_{\mathfrak{F}} \stackrel{\text { def }}{=}\left\{\mathscr{B} \subset \mathscr{X}_{\mathfrak{F}} \mid \quad \forall s \in S: \mathscr{B}^{s} \in \mathfrak{B}(\mathfrak{I}(k))\right\}, \\
\mathcal{N}_{\mathfrak{F}} \stackrel{\text { def }}{=}\left\{\mathscr{Z} \in \mathfrak{M}_{\mathfrak{F}} \mid \quad \forall s \in S: \mathscr{B}^{s} \in \mathcal{N}\left(\mu_{s}\right)\right\}
\end{gathered}
$$

and let $\Phi_{\mathfrak{F}}: \mathscr{X}_{\mathfrak{F}} \rightarrow \mathfrak{F}$ be the projection onto the first coordinate. Then $\left(\mathscr{X}_{\mathfrak{F}}, \mathfrak{M}_{\mathfrak{F}}, \mathcal{N}_{\mathfrak{F}}\right)$ is a multi-standard measurable space with nullity and $\Phi_{\mathfrak{F}}$ is a covering such that $\mathcal{J}\left(\Phi_{\mathfrak{F}}\right)=\mathcal{J}[\mathfrak{F}]$.

The details of the proof are left to the reader.

Below we give a few illustrative examples of ideals of the form $\mathcal{J}[\mathfrak{F}]$. Recall that $k$ denotes the length of tuples of operators. Below we (naturally) identify subsets of $\mathbb{C}$ with subsets of $\mathfrak{I}_{1}(1)$.

- $\mathfrak{J}[\mathbb{C}]$ is the ideal of single normal operators;

- $\mathcal{J}[\mathbb{R}]$ is the ideal of single selfadjoint operators;

- $\mathcal{J}[\mathbb{T}]$ is the ideal of single unitary operators ( $\mathbb{T}$ is the unit circle);

- $\mathcal{J}\left[\mathfrak{I}_{1}(k)\right]$ is the ideal of $k$-tuples of commuting normal operators;

- $\mathcal{J}[\mathfrak{I}(k)]=\mathcal{J}_{I}^{f}$.

The issue we want to focus on here is the problem of p-isomorphicity of ideals. To this end, we introduce

4.13. Definition. Let $\mathcal{J}$ and $\mathcal{J}$ be two finite type $I$ ideals of unitary equivalence classes of, respectively, $k$-tuples and $\ell$-tuples of operators. $\mathcal{J}$ and $\mathcal{J}$ are said to be $p$-isomorphic, if there exists a bijection $\Psi: \mathcal{J} \rightarrow \mathcal{J}$ such that:

(pI1) for every nonempty collection $\left\{\mathrm{X}_{s}\right\}_{s \in S}$ of members of $\mathcal{J}, \Psi\left(\bigoplus_{s \in S} \mathrm{X}_{s}\right)=$ $\bigoplus_{s \in S} \Psi\left(\mathrm{X}_{s}\right)$

(pI2) for any $\mathrm{X} \in \mathcal{J}, \Psi(\mathrm{X}) \in \mathfrak{I}(\ell) \Longleftrightarrow \mathrm{X} \in \mathfrak{I}(k)$;

(pI3) there exists a covering $\Phi: \mathscr{X} \rightarrow \mathfrak{I}(k)$ with $\mathcal{J}(\Phi)=\mathcal{J}$ such that:

- $\Psi \circ \Phi: \mathscr{X} \rightarrow \mathfrak{I}(\ell)$ is a covering with $\mathcal{J}(\Psi \circ \Phi)=\mathcal{J}$;

- for every cardinal-valued measurable function $m: \mathscr{X} \rightarrow$ Card,

$$
\Psi\left(\bigoplus_{x \in \mathscr{X}}^{\mathcal{N}} m(x) \odot \Phi(x)\right)=\bigoplus_{x \in \mathscr{X}}^{\mathcal{N}} m(x) \odot(\Psi \circ \Phi)(x) .
$$

In the above situation, $\Psi$ is said to be a p-isomorphism. 
The concept of p-isomorphisms was introduced in [10 (see $\S 6.3 .3$ there). Two ideals which are p-isomorphic have similar structures induced by the direct sum operation and, in a sense, the same Borel and spectral complexities (here 'spectral' refers to properties expressible by means of coverings). Roughly speaking, whatever can be said about one of them in terms of direct sums and coverings, it has its natural counterpart for the other ideal.

Our main result on p-isomorphic ideals is formulated below.

4.14. Theorem. If $\mathfrak{F} \subset \mathfrak{I}(k)$ and $\mathfrak{G} \subset \mathfrak{I}(\ell)$ are two Borel sets of the same cardinality, then the ideals $\mathcal{J}[\mathfrak{F}]$ and $\mathcal{J}[\mathfrak{G}]$ (of unitary equivalence classes of $k$-tuples and $\ell$-tuples, respectively) are p-isomorphic. In particular, $\mathfrak{J}[\mathfrak{F}]$ is p-isomorphic to the ideal $\mathfrak{J}[\mathbb{T}]$ of all single unitary operators provided $\mathfrak{F}$ is uncountable.

Proof. Since $\mathfrak{F}$ and $\mathfrak{G}$ are standard Borel spaces of the same cardinality, there exists a Borel isomorphism $\psi: \mathfrak{F} \rightarrow \mathfrak{G}$ between them. Let $\mathscr{M} \stackrel{\text { def }}{=} \mathscr{M}_{\mathfrak{F}}=\left\{\mu_{s}\right\}_{s \in S}$, $(\mathscr{X}, \mathfrak{M}, \mathcal{N}) \stackrel{\text { def }}{=}\left(\mathscr{X}_{\mathfrak{F}}, \mathfrak{M}_{\mathfrak{F}}, \mathcal{N}_{\mathfrak{F}}\right)$ and $\Phi \stackrel{\text { def }}{=} \Phi_{\mathfrak{F}}: \mathscr{X} \rightarrow \mathfrak{F}$ be as in Proposition 4.12 (applied for $\mathfrak{F})$. For each $s \in S$ let $\nu_{s}: \mathfrak{B}(\mathfrak{I}(\ell)) \rightarrow[0,1]$ be the transport of $\mu_{s}$ under $\psi$. Then $\mathscr{M}_{\mathfrak{G}} \stackrel{\text { def }}{=}\left\{\nu_{s}\right\}_{s \in S}$ is a maximal collection of mutually singular Borel probabilistic measures on $\mathfrak{I}(\ell)$ which are supported on $\mathfrak{G}$, since $\psi$ is a Borel isomorphism. Now starting with $\mathscr{M}_{\mathfrak{G}}$, we build $\left(\mathscr{X}_{\mathfrak{G}}, \mathfrak{M}_{\mathfrak{G}}, \mathcal{N}_{\mathfrak{G}}\right)$ and $\Phi_{\mathfrak{G}}: \mathscr{X}_{\mathfrak{G}} \rightarrow \mathfrak{G}$ as in Proposition 4.12 (with $\mathfrak{G}$ in place of $\mathfrak{F})$. Then $\Phi_{\mathfrak{G}}$ is a covering such that $\mathcal{J}\left(\Phi_{\mathfrak{G}}\right)=\mathcal{J}[\mathfrak{G}]$. Let $\Psi_{0}: \mathscr{X} \rightarrow \mathscr{X}_{\mathfrak{G}}$ be given by $\Psi_{0}(\mathrm{~T}, s)=(\psi(\mathrm{T}), s)$. It is easy to check that $\Psi_{0}$ is a bijection such that $\mathfrak{M}_{\mathfrak{G}}=\left\{\Psi_{0}(\mathscr{B}): \mathscr{B} \in \mathfrak{M}\right\}$ and $\mathcal{N}_{\mathfrak{G}}=\left\{\Psi_{0}(\mathscr{Z}): \quad \mathscr{Z} \in \mathcal{N}\right\}$. So, $\Psi_{0}$ is an isomorphism between measurable spaces with nullities. We therefore conclude that $\Phi_{\mathfrak{G}} \circ \Phi_{0}$ is a covering for $\mathcal{J}[\mathfrak{G}]$. But $\Phi_{\mathfrak{G}} \circ \Psi_{0}=\psi \circ \Phi$. We now define $\Psi: \mathcal{J}[\mathfrak{F}] \rightarrow \mathcal{J}[\mathfrak{G}]$ by the rule:

$$
\Psi\left(\bigoplus_{x \in \mathscr{X}}^{\mathcal{N}} m(x) \odot \Phi(x)\right) \stackrel{\text { def }}{=} \bigoplus_{x \in \mathscr{X}}^{\mathcal{N}} m(x) \odot \psi(\Phi(x))
$$

where $m: \mathscr{X} \rightarrow$ Card is a cardinal-valued measurable function. Item (C) of Theorem 4.6 implies that: the definition of $\Psi$ is complete and correct; $\Psi$ is a bijection; and $\Psi(X \oplus Y)=\Psi(X) \oplus \Psi(Y)$ for any $X, Y \in \mathcal{J}[\mathfrak{F}]$. So, it follows from Theorem 6.1.4 in 10 that $\Psi$ satisfies condition $(\mathrm{pI1})$. Moreover, $\Psi(\mathrm{X})=\psi(\mathrm{X})$ for each $\mathrm{X} \in \mathfrak{F}$, which yields (pI2) and (pI3). Now the note that each uncountable Borel set in $\mathfrak{I}(k)$ has the same cardinality as $\mathbb{T}$ completes the proof.

We conclude the paper with a note concerning multiplicity theory for tuples of operators affiliated with finite type I von Neumann algebras.

4.15. Remark. Based on the results obtained in the paper, one may easily extend, in a transparent way, multiplicity theory for normal operators to arbitrary (finite tuples of) operators which are affiliated with finite type I von Neumann algebras. This idea may be realised as follows. Let $\mathscr{M}=\left\{\mu_{s}\right\}_{s \in S},(\mathscr{X}, \mathfrak{M}, \mathcal{N})$ and $\Phi: \mathscr{X} \rightarrow$ $\mathfrak{I}(k)$ be as in Proposition 4.8. For $\mathrm{X} \in \mathcal{J}_{I}^{f}$ we use $m_{\mathrm{X}}$ to denote the multiplicity function of $\mathbf{X}$ (relative to $\Phi$ ). Now if $\boldsymbol{T}=\left(T_{1}, \ldots, T_{k}\right)$ acts in a separable Hilbert space and its unitary equivalence class $\mathrm{T}$ belongs to $\mathcal{J}_{I}^{f}$, then there is a standard set $\mathscr{B} \subset \mathscr{X}$ such that $m_{\mathrm{\top}}(x)=0$ for $\mathcal{N}$-almost all $x \in \mathscr{X} \backslash \mathscr{B}$ and $m_{\mathrm{\top}}(x) \leqslant \aleph_{0}$ for each $x \in \mathscr{B}$ (see e.g. item (h) in Theorem 5.6.14 in [10]). These imply that we may think of $m_{\mathrm{T}}$ as of a function with values in $\{0,1,2, \ldots, \infty\}$. Moreover, since $\mathscr{B}$ is standard, the set $S_{0}=\left\{s \in S: \mathscr{B}^{s} \notin \mathcal{N}\left(\mu_{s}\right)\right\}$ is countable (finite or not), say $S_{0}=\left\{s_{n}: 1 \leqslant n<N\right\}$ (where $N$ is finite or not). Further, we can find a collection $\left\{\mathfrak{S}_{n}: 1 \leqslant n<N\right\}$ of pairwise disjoint Borel subsets of $\mathfrak{I}(k)$ such that $\mu_{s_{n}}\left(\mathfrak{S}_{n}\right)=1$ whenever $n<N$. Now define a measure $\mu_{\boldsymbol{T}}: \mathfrak{B}(\mathfrak{I}(k)) \rightarrow[0,1]$ as $\mu_{\boldsymbol{T}} \stackrel{\text { def }}{=} \sum_{n<N} \frac{1}{2^{n}} \mu_{s_{n}}$ and a function $m_{\boldsymbol{T}}: \mathfrak{I}(k) \rightarrow\{0,1,2, \ldots, \infty\}$ by the rules: 
$m_{\boldsymbol{T}}(\mathbf{X})=m_{\mathrm{T}}\left(\mathbf{X}, s_{n}\right)$ for $\mathrm{T} \in \mathfrak{S}_{n}($ with $n<N)$ and $m_{\mathrm{\top}}(\mathbf{X})=0$ for $\mathbf{X} \notin \bigcup_{n<N} \mathfrak{S}_{n}$. In this way to each $k$-tuple $\boldsymbol{T}$ we have assigned a finite Borel measure $\mu_{\boldsymbol{T}}$ on $\mathfrak{I}(k)$ and a measurable function $m_{\boldsymbol{T}}: \mathfrak{I}(k) \rightarrow\{0,1,2, \ldots, \infty\}$. One may show that two $k$-tuples $\boldsymbol{T}$ and $\boldsymbol{S}$ (acting in separable Hilbert spaces) are unitarily equivalent iff the measures $\mu_{\boldsymbol{T}}$ and $\mu_{\boldsymbol{S}}$ are mutually absolutely continuous and $m_{\boldsymbol{T}}(\mathbf{X})=m_{\boldsymbol{S}}(\mathbf{X})$ for $\mu_{\boldsymbol{T}}$-almost all $\mathbf{X} \in \mathfrak{I}(k)$. The details are left to the reader.

\section{REFERENCES}

[1] H. Becker and A.S. Kechris, The Descriptive Set Theory of Polish Group Actions (London Math. Soc. Lecture Note Series, vol. 232), University Press, Cambridge, 1996.

[2] A. Brown, C.-K. Fong, D.W. Hadwin, Parts of operators on Hilbert space, Illinois J. Math. 22 (1978), 306-314.

[3] C. Castaing, Quelques problèmes de mesurabilité liées à la théorie de la commande, C. R. Paris 262 (1966), 409-411.

[4] J.B. Conway, A Course in Functional Analysis (Graduate Texts in Mathematics, vol. 96), Springer, New York, 1990.

[5] J. Dixmier, $C^{*}$-algebras, North-Holland Publ. Co., Amsterdam, 1977.

[6] J. Ernest, Charting the operator terrain, Mem. Amer. Math. Soc. 171 (1976), 207 pp.

[7] A.S. Kechris, Classical Descriptive Set Theory (Graduate Texts in Mathematics, Volume 156), Springer-Verlag, New York, 1995.

[8] K. Kuratowski and A. Mostowski, Set Theory with an Introduction to Descriptive Set Theory, PWN - Polish Scientific Publishers, Warszawa, 1976.

[9] R. Longo, Solution of the factorial Stone-Weierstrass conjecture. An application of the theory of standard split $W^{*}$-inclusions, Invent. Math. 76 (1984), 145-155.

[10] P. Niemiec, Unitary equivalence and decompositions of finite systems of closed densely defined operators in Hilbert spaces, Dissertationes Math. (Rozprawy Mat.) 482 (2012), 1-106.

[11] P. Niemiec, Elementary approach to homogeneous $C^{*}$-algebras, to appear (http://arxiv.org/abs/1203.0857).

[12] S. Popa, Semiregular maximal abelian *-subalgebras and the solution to the factor state Stone-Weierstrass problem, Invent. Math. 76 (1984), 157-161.

[13] J. von Neumann, On Rings of Operators. Reduction Theory, Ann. Math. 50 (1949), 401-485.

Instytut Matematyki, Wydzią Matematyki i Informatyki, Uniwersytet Jagielloński, Ul. LOJASIEWicza 6, 30-348 Kraków, POLAND

E-mail address: piotr.niemiec@uj.edu.pl 